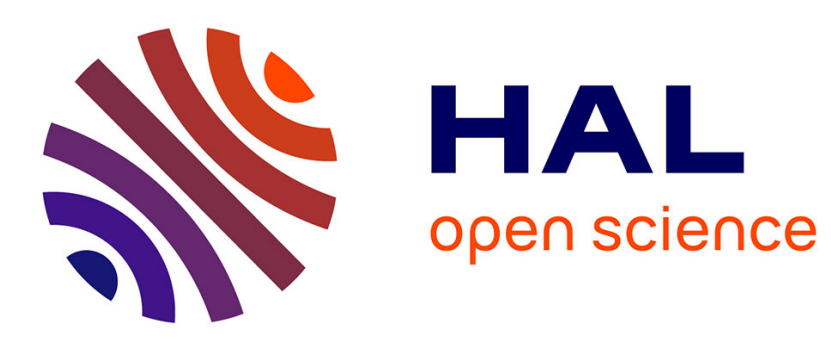

\title{
First data from the ANTARES neutrino telescope
}

\author{
V. Bertin
}

\section{To cite this version:}

V. Bertin. First data from the ANTARES neutrino telescope. Neutrino 2006, Jun 2006, Santa Fe, United States. pp.32-36, 10.1016/j.nuclphysbps.2011.03.088 . in2p3-00466984

\section{HAL Id: in2p3-00466984 https://hal.in2p3.fr/in2p3-00466984}

Submitted on 9 Apr 2014

HAL is a multi-disciplinary open access archive for the deposit and dissemination of scientific research documents, whether they are published or not. The documents may come from teaching and research institutions in France or abroad, or from public or private research centers.
L'archive ouverte pluridisciplinaire HAL, est destinée au dépôt et à la diffusion de documents scientifiques de niveau recherche, publiés ou non, émanant des établissements d'enseignement et de recherche français ou étrangers, des laboratoires publics ou privés. 


\title{
First data from the ANTARES neutrino telescope
}

\author{
V. Bertin ${ }^{1}$ on behalf of the ANTARES Collaboration \\ ${ }^{1}$ C.P.P.M., CNRS/IN2P3 et Université de la Méditerrannée, \\ Case 902, 163, avenue de Luminy, 13288 Marseille cedex 9, France \\ E-mail: bertin@cppm.in2p3.fr
}

\begin{abstract}
This contribution reviews the recent progress achieved towards building the ANTARES neutrino telescope. The first results obtained by the operation of a Mini Instrumentation Line with Optical Modules, "MILOM", and the first complete detector line are highlighted.
\end{abstract}

\section{The ANTARES detector}

The European Collaboration ANTARES aims at building and operating a large undersea neutrino telescope located at a depth of $2500 \mathrm{~m}$ in the Mediterranean Sea, offshore from Toulon in France [1]. Neutrinos will be detected through their interaction in the matter surrounding the detector, producing muons radiating Cherenkov light while propagating in the sea water. Photons are recorded by a lattice of Optical Modules (OMs) [2], consisting of 10" hemispherical photomultiplier tubes (PMTs) [3] housed in pressure resistant glass spheres, installed on a set of mooring lines. The reconstruction of the muon track direction, pointing to a fraction of a degree towards the direction of the parent neutrino source for high energy neutrinos, is achieved from the measurement of the arrival times of the Cherenkov photons on the OMs, as well as their position in space.

The complete ANTARES detector will consist of 12 lines of 25 storeys, each storey being equipped with a triplet of Optical Modules looking at $45^{\circ}$ downward and an electronics container mounted on a titanium frame, giving a grand-total of 900 OMs. On each storey, the local electronics container includes the front-end electronics of the PMTs, an Ethernet board for the data acquisition and the detector Slow Control, electronics boards for clock distribution and for Dense Wavelength Division Multiplexing of the Ethernet transmission, and a tiltmeter-compass board measuring the local tilt and orientation of the storey. Some storeys also support a hydrophone for acoustic positioning or an LED Optical Beacon used for inter-string time calibration.

The vertical distance between storeys is $14.5 \mathrm{~m}$, the first one being placed at $100 \mathrm{~m}$ above the sea bed, leading to a total height of the detector strings of $480 \mathrm{~m}$. Each string is anchored on the sea floor at a distance of $70 \mathrm{~m}$ from its neighbours. Every line is individually connected to a Junction Box by an interconnection cable a few hundred metres long. The Junction Box is itself linked to the shore by a 40 $\mathrm{km}$ long electro-optical cable equipped with 48 optical fibres.

The construction of the ANTARES detector started in October 2001 with the deployment of the main electro-optical cable from the ANTARES site, located in the Mediterranean Sea $\left(42^{\circ} 48^{\prime} \mathrm{N}\right.$ $6^{\circ} 10^{\prime} \mathrm{E}$ ) offshore Toulon (France) at a depth of $2475 \mathrm{~m}$, to the beach of La Seyne-sur-Mer where the shore station of the experiment is situated. In December 2002, the Junction Box was connected at the end of this cable and immersed on the site. In Spring 2003, two small test lines, the Prototype Sector 
Line (PSL) and the Mini Instrumentation Line (MIL), were installed, connected and operated for a few months. The genuine operation of the ANTARES neutrino telescope really started in April 2005 after the connection of the Mini Instrumentation Line with Optical Modules (MILOM) and more recently the installation of the first full complete detector line, Line 1, in March 2006. The second line, Line 2, was in integration phase in June 2006, at the time of the Neutrino'06 Conference, it has been successfully deployed on the ANTARES site at the end of July 2006 as scheduled. Thanks to two assembly sites running in parallel, the ANTARES detector is foreseen to be fully deployed and operational by the end of 2007 for several years of physics data taking.

The main performance parameters expected for the complete ANTARES neutrino telescope are summarized in figure 1. The left plot shows the effective area for neutrinos as a function of the neutrino energy for various incident angles. The effective area reaches $1 \mathrm{~m}^{2}$ for $\mathrm{E}_{\mathrm{v}}>100 \mathrm{TeV}$, the energy above which the Earth shadowing starts to be of some importance. The right plot shows the angular resolution for the reconstructed muon compared to the true muon direction and to the parent neutrino direction, as a function of the neutrino energy. While the angular resolution is dominated by the physical angle between the muon and the parent neutrino at low energy, it is dominated by the reconstruction for $E_{v} \geq 10 \mathrm{TeV}$ and is expected to be as good as $0.3^{\circ}$. In this regime, the angular resolution is mainly dominated by two effects: the scattering and the chromatic dispersion of the Cherenkov light during its travel into the sea water, contributing for a time arrival spread of $\sigma \sim 1 \mathrm{~ns}$; the transit time spread (TTS) of the PMT signals being $\sigma \sim 1.3 \mathrm{~ns}$.

To achieve this good angular resolution, the ANTARES detector is designed such as additional electronics contributions to the time calibration contribute for less than $\sim 0.5 \mathrm{~ns}$ to the time-stamping of the detected photons. In addition, the relative position reconstruction of the OM has to be controlled with a precision of $\sim 10 \mathrm{~cm}$.
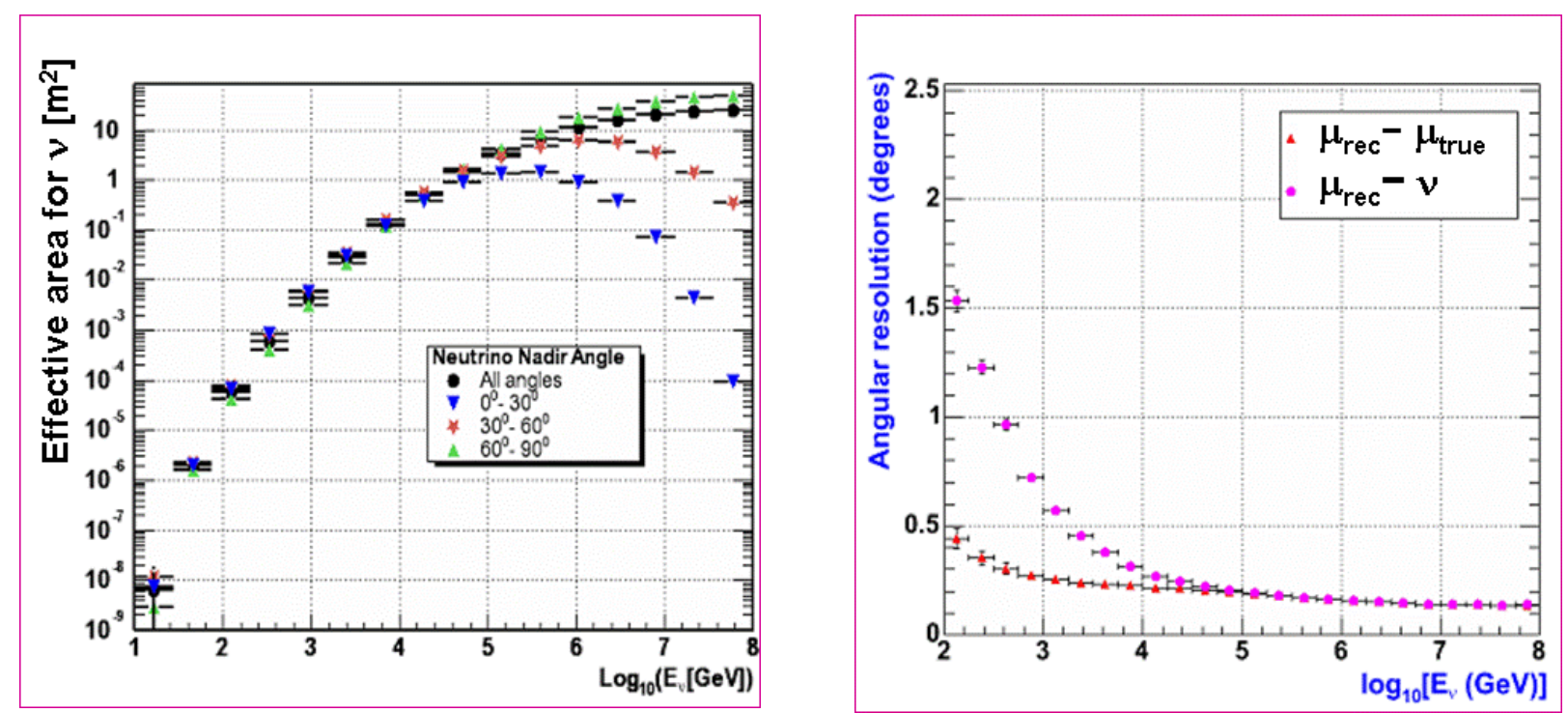

Figure 1. Expected performance for the complete 12 lines ANTARES neutrino telescope. The left figure shows the effective area for neutrinos as a function of the neutrino energy for several incident angles. The right figure shows the angular resolution of the reconstructed muon compared to the true muon direction and to the parent neutrino as a function of the neutrino energy.

\section{Results from the first ANTARES lines}

\subsection{The MILOM line}

The current data taking of the ANTARES detector started in March 2005 with the operation of the MILOM [4]. This instrumentation line, partly devoted to multi-disciplinary and environmental studies, 
consists of an instrumented releasable anchor, the Bottom String Socket (BSS), and three storeys respectively located at $100 \mathrm{~m}, 117 \mathrm{~m}$ and $169 \mathrm{~m}$ above the sea bed. The middle storey is a standard ANTARES storey housing a triplet of Optical Modules. The main other devices are a water current profiler located on the top storey, an LED Optical Beacon held on the bottom storey, an acoustic positioning transducer attached to the BSS and a seismometer buried into the sea floor $50 \mathrm{~m}$ away from the MILOM.

2.1.1. Optical background measurements. The operation of the MILOM allowed a continuous monitoring of the background rates of the Optical Modules. A typical OM counting rate display exhibits a baseline of $\sim 60-80 \mathrm{kHz}$ largely dominated by optical background due to ${ }^{40} \mathrm{~K}$ decays and bioluminescence activities coming from bacteria, as well as bursts of a few seconds duration produced by bioluminescent emission of macro-organisms [5]. Figure 2 (left) shows the baseline rates recorded by the three OMs of the MILOM during a period of three months in Autumn 2005. A seasonal variation of the bioluminescence component of the baseline is clearly observed. The $15 \%$ higher counting rate of OM1 during the full period is due to a lower threshold set on the readout of this Optical Module. Figure 2 (right) shows the burstfraction, defined as the fraction of the time when the counting rate is higher than the baseline by $20 \%$ during a 15 min interval, as a function of the water current intensity. A strong correlation of these two quantities is clearly observed.
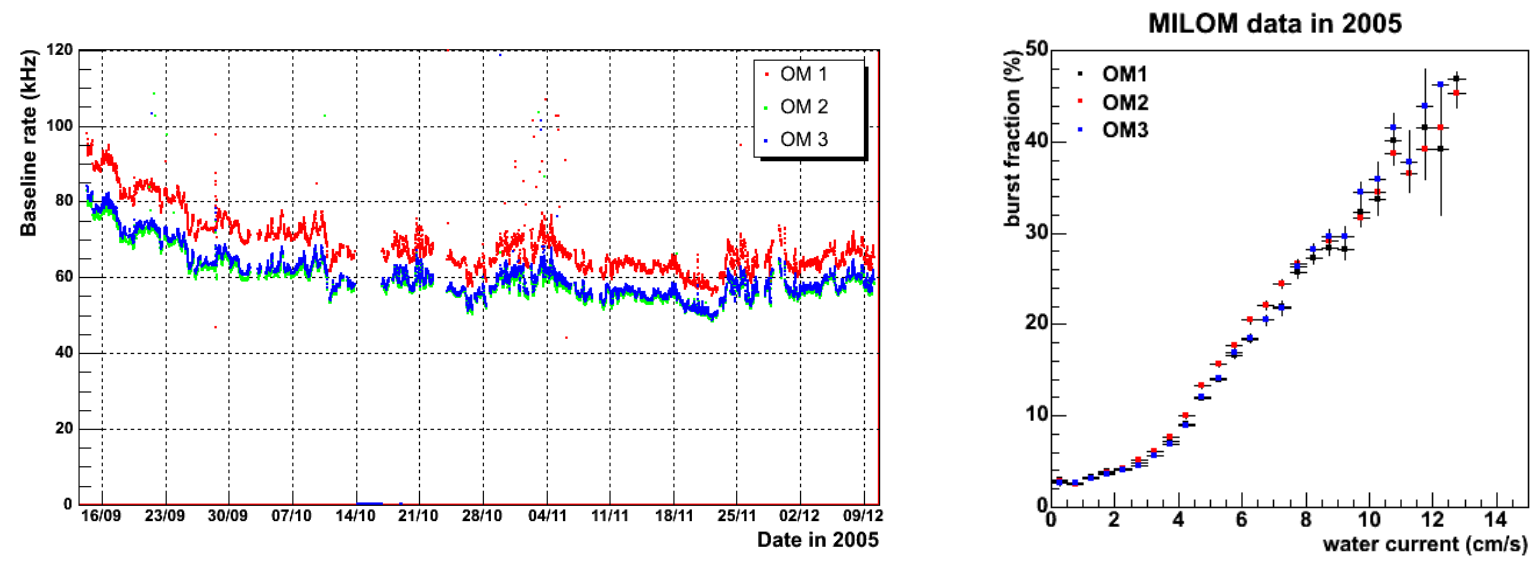

Figure 2. Baseline rates recorded by the three OMs of the MILOM during Autumn 2005 (left) and burstfraction as function of the water current intensity (right).

The time coincidences between pairs of neighbouring Optical Modules have also been studied. These distributions exhibit a flat background of random coincidences and a Gaussian peak of few ns width due to genuine coincidences of ${ }^{40} \mathrm{~K}$ radioactive decays producing two detected photons. The ${ }^{40} \mathrm{~K}$ coincidence rate is measured to be $13.0 \pm 0.5 \mathrm{~Hz}$ and is in good agreement with a simulation the signals induced by ${ }^{40} \mathrm{~K}$ decays which leads to a coincidence rate of $12 \mathrm{~Hz}$ with a $4 \mathrm{~Hz}$ systematic error due to uncertainties in the effective area and angular response of the OMs.

2.1.2. Time calibration with the LED Optical Beacon. The time calibration of the MILOM Optical Modules has been checked by flashing the LED Optical Beacon located on the bottom storey. This device consists of a glass cylinder container containing 36 blue LEDs synchronised in time in order to produced intense light flashes with a time dispersion $<0.5 \mathrm{~ns}$. The time calibration of the OMs can be checked either by looking at the arrival time of the signal on the PMT relative to the time of the flash, or by the time difference of the flash arrival time measured by two adjacent OMs. Due to the large intensity of the light flashes and the short $15 \mathrm{~m}$ distance of propagation of the light into the water, the time stamping of the OM signal is dominated by its electronics contribution and not by the TTS of the 
PMT in this case. The measured distributions confirm that the electronics contribution to the time calibration is $\leq 0.5 \mathrm{~ns}$ as expected.

\subsection{The Line 1}

The first complete line of the ANTARES neutrino telescope, Line 1, has been deployed on the site on February $14^{\text {th }} 2006$ and connected two weeks later on March $2^{\text {nd }}$ by using the Remote Operated Vehicle Victor of IFREMER. This line is made of a BSS and of 25 storeys, holding a total of 75 OMs. It also includes 4 LED Optical Beacons and 5 acoustic positioning hydrophones spread along the line, as well as an acoustic transducer on its BSS.

2.2.1. Time calibration with the MILOM LED Optical Beacon. The time calibration of the Line 1 OMs can also be checked by flashing the LED Optical Beacon located on the MILOM bottom storey. Figure 3 (left) shows the detection time spread of the LED Optical Beacon flashes by an OM of Line 1 located on a storey at the same altitude than the MILOM LED Optical Beacon. In this case, the measured distribution width is $\sigma=0.7 \mathrm{~ns}$ for a "horizontal" travel path of $\sim 80 \mathrm{~m}$ of the light in the sea water. Figure 3 (right) shows in comparison the detection time spread for an OM of Line 1 located at a higher altitude corresponding to a "diagonal" travel path of the flash light of $\sim 150 \mathrm{~m}$. A wider distribution is clearly observed due to the smaller intensity of the detected signal, as well as a tail of delayed photons coming from scattering light. All measured distributions have been found in good agreement with expectations.
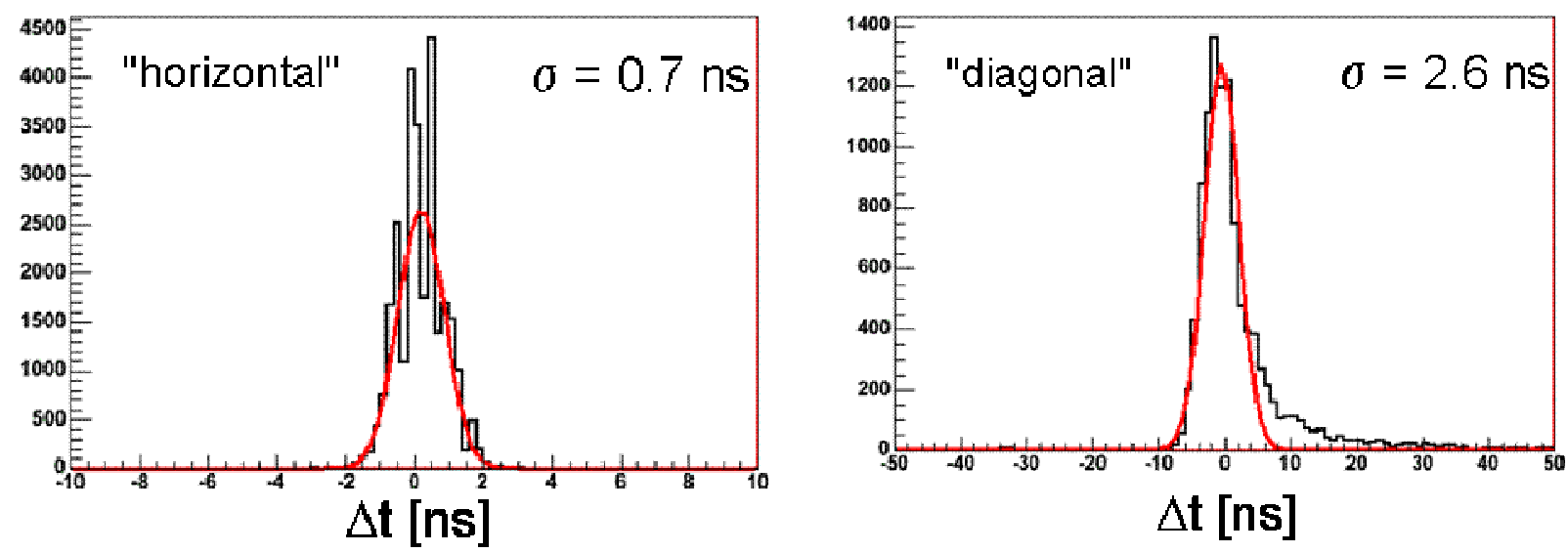

Figure 3. Detection time spread of the MILOM LED Optical Beacon flashes by an OM of Line 1 located at the same altitude as the LED Optical Beacon at an "horizontal" distance of $\sim 80 \mathrm{~m}$ (left) and by an $\mathrm{OM}$ at a higher altitude at a "diagonal" distance of $\sim 150 \mathrm{~m}$ (right).

2.2.2. Acoustic positioning measurements. The reconstruction of the detector geometry in real time is primarily based on acoustic triangulation of a small number of hydrophones scattered along every line. The triangulation is performed from distance measurements of each hydrophone to several fixed acoustic emitters located either on every line anchor base (transducers) or on autonomous pyramidal structures anchored around the detector field (transponders). A relative positioning of the hydrophones in space with a precision of few $\mathrm{cm}$ is necessary in order to obtain a precision of $\sim 10 \mathrm{~cm}$ on the $\mathrm{OM}$ positions as a result of the line shape reconstruction performed by the addition of the tilts and heading measurements of every storey. The concomitant operation of MILOM and Line 1 has allowed a check of the acoustic system performance by performing the Line 1 hydrophone triangulation based on the acoustic emission of the MILOM transducer and two autonomous transponders. The good resolution of the acoustic system, found to be well within the specification, can be appreciated on figure 4 which shows the radial displacement of the lowest hydrophone of Line 1, located on its bottom storey at 100 $\mathrm{m}$ above the sea bed, with respect to the line axis during a period of two weeks. 


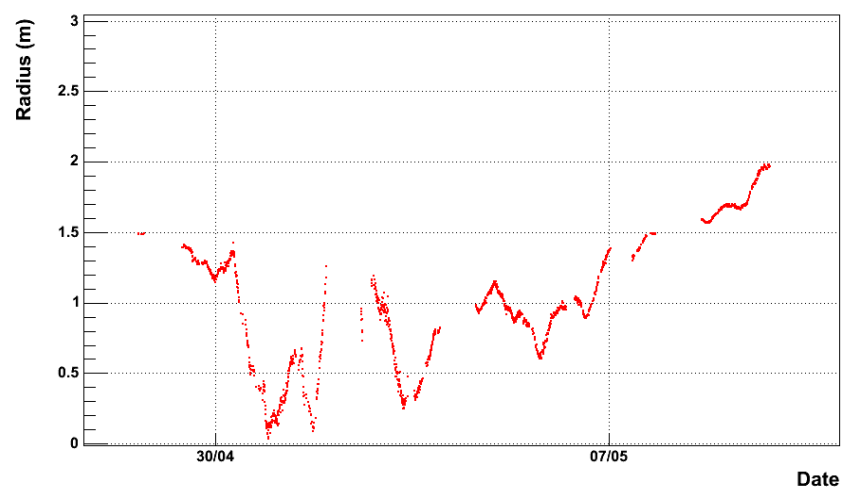

Figure 4. Radial displacement of the Line 1 lowest hydrophone with respect to the line axis measured by the acoustic relative positioning system during a period of two weeks.

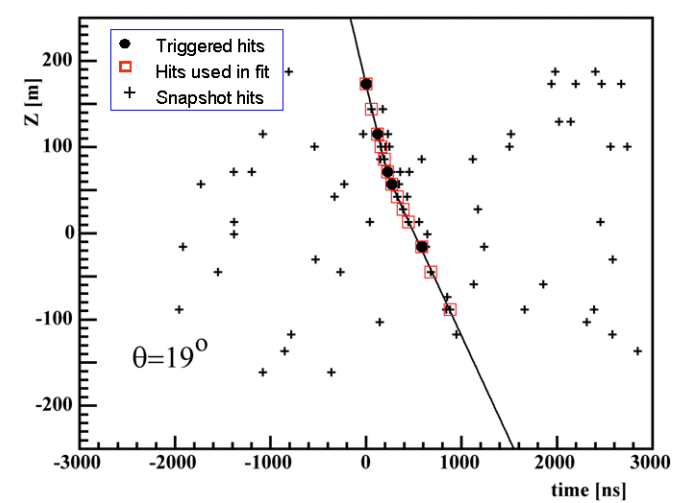

Figure 5. Example of a downward-going atmospheric muon track reconstruction with the ANTARES Line 1.

2.2.3. Reconstruction of atmospheric muons. Although the OMs point at $45^{\circ}$ downwards, the ANTARES detector has a none negligible efficiency for the detection and the reconstruction of downward-going atmospheric muons. The event selection is first performed by an online filter algorithm, running at the shore station, which looks for a set of $\geq 4$ local coincidence hits, the triggered hits, causally compatible with a muon track passing through the detector during a $4 \mu$ s time window. The muon reconstruction is then performed with a $\chi^{2}$ fit of the hit times as function of their altitudes, to a hyperbola corresponding to the intersection of the muon Cherenkov light front with the line plane, in order to determine the zenith angle of the muon track. An example of such a muon track fit is shown on figure 5. Several thousand of atmospheric muons have already been reconstructed after a few weeks of operation of the Line 1, the first being detected only two days after its connection. The study of the muon angular distribution is in progress. The preliminary results already show that the muon reconstruction is working well and that the hunt for the first undersea neutrino can be started.

\section{Conclusion}

The ANTARES Collaboration has made a major step forward during the last year by the operation of a Mini Instrumentation Line with Optical Modules, the MILOM, for more than a year, and the installation of the first complete line of the detector in Spring 2006. All studies performed with these two lines show that the detector behaves well within the design specification and that all technical problems are solved. The detector should be fully installed by the end of 2007 and in operation for science during at least five years. It is also considered as a milestone towards the building of a $\mathrm{km}^{3}$ underwater detector for which a design study is under preparation.

\section{References}

[1] Aslanides E et al., ANTARES Collaboration 1999 the ANTARES Proposal Preprint astro$\mathrm{ph} / 9907432$

[2] Amram P et al. ANTARES Collaboration 2002 Nucl. Inst. Meth. A 484369 (Preprint astro$\mathrm{ph} / 0112172)$

[3] Aguilar J A et al. ANTARES Collaboration 2005 Nucl. Inst. Meth. A 555132 (Preprint physics /0510031)

[4] Aguilar J A et al. ANTARES Collaboration 2006 First results of the Instrumentation Line for the deep-sea ANTARES neutrino telescope Preprint astro-ph/0606229

[5] Amram P et al. ANTARES Collaboration 2000 Astropart. Phys. 13127 (Preprint astro$\mathrm{ph} / 9910170)$ 